\title{
The unique prognostic characteristics of tumor deposits in colorectal cancer patients
}

\author{
Fangqi Liu ${ }^{1,2 \#}$, Jiang Zhao ${ }^{3 \#}$, Cong $\mathrm{Li}^{1,2}$, Yuchen $\mathrm{Wu}^{1,2}$, Wang Song ${ }^{1,2}$, Tianan Guo ${ }^{1,2}$, Shiqing Chen ${ }^{4}$, \\ Sanjun Cai ${ }^{1,2}$, Dan Huang ${ }^{1,5}$, Ye $\mathrm{Xu}^{1,2}$ \\ ${ }^{1}$ Department of Colorectal Surgery, Fudan University Shanghai Cancer Center, Shanghai 200032, China; ${ }^{2}$ Department of Oncology, Shanghai \\ Medical College, Fudan University, Shanghai 200032, China; ${ }^{3}$ Department of General Surgery, Xuhui District Central Hospital of Shanghai, \\ Shanghai 200031, China; ${ }^{4}$ The Medical Department, 3D Medicines Inc., Shanghai 201114, China; ${ }^{5}$ Department of Pathology, Shanghai Medical \\ College, Fudan University, Shanghai 200032, China \\ Contributions: (I) Conception and design: Y Xu, D Huang, F Liu, J Zhao, S Cai; (II) Administrative support: Y Xu, D Huang; (III) Provision of \\ study materials or patients: Y Xu, D Huang, S Cai; (IV) Collection and assembly of data: F Liu, J Zhao, C Li, Y Wu, T Guo; (V) Data analysis and \\ interpretation: F Liu, J Zhao, S Chen, C Li, Y Wu, T Guo; (VI) Manuscript writing: All authors; (VII) Final approval of manuscript: All authors. \\ \#These authors contributed equally to this work. \\ Correspondence to: Ye Xu. Department of Colorectal Surgery, Fudan University Shanghai Cancer Center, 270 Dong An Road, Shanghai 200032, \\ China. Email: xuye021@163.com; Dan Huang. Department of Pathology, Fudan University Shanghai Cancer Center, 270 Dong An Road, Shanghai \\ 200032, China. Email: dianehuangfdcc@gmail.com.
}

Background: Both AJCC 7th and 8th TNM systems have included tumor deposits (TDs) in nodal staging when lymph nodes metastases (LNMs) are negative in colorectal cancer (CRC). However, the prognostic role of TDs has not been determined in the presence of positive LNMs.

Methods: Two independent large-scale cohorts of CRC patients from the Surveillance Epidemiology and End Results (SEER) database $(n=69,178)$ [2010-2013] and Fudan University Shanghai Cancer Center (FUSCC) (n=3,137) [2010-2014] were retrospectively analyzed. Kaplan-Meier method was used to estimate survival curves and univariate and multivariate analyses were performed by Cox proportional hazard model.

Results: TDs were observed in $12.3 \%(n=8,480)$ and $14.8 \%(n=463)$ of patients in the SEER and FUSCC cohorts, respectively. Multivariate analysis suggested TDs were an independent adverse prognostic factors for overall survival (OS) $(\mathrm{P}<0.001)$. Remarkably, both cohorts showed the presence of TDs was significantly associated with OS, but not was the number of TDs $(\mathrm{P}=0.982$ and $\mathrm{P}=0.252$ for the SEER and FUSCC cohorts, respectively). In the presence of LNMs, positive TDs were associated with a shorter OS [hazard ratio (HR): 2.69, 95.0\% confidence interval (CI): 2.597-2.778; $\mathrm{P}<0.001]$. Further analysis combining TDs with LNMs demonstrated that the prognosis of patients with N1TD (N1 with positive TDs) was same as the $\mathrm{N} 2$ patients, and N2TD (N2 with positive TDs) patients had much worse prognosis than $\mathrm{N} 2(\mathrm{P}<0.001)$.

Conclusions: Our results have shown the unique features of TDs in patients with CRC, different from LNMs. In the presence of LNMs, TDs should also be considered in TMN system.

Keywords: Tumor deposits (TDs); colorectal cancer (CRC); prognosis; lymph nodes metastasis (LNM); TNM system

Submitted Sep 02, 2019. Accepted for publication Nov 01, 2019.

doi: $10.21037 /$ atm.2019.11.69

View this article at: http://dx.doi.org/10.21037/atm.2019.11.69 


\section{Introduction}

Colorectal cancer (CRC), as one of the most common malignancy, is the 5 th most common cause of death in China (1). In spite of increase in the incidence of CRC, its mortality rate is decreasing in China last almost 20 years (2). Overall increase in CRC survival benefits from more detailed staging systems and more treatment choices. The TNM staging system, which plays a crucial role in cancer treatment, is applied worldwide for various tumor types, including CRC. In order to determine prognostic outcomes and guide treatment choices more precisely, the TNM staging system keeps pace with the times.

In recent editions of The American Joint Committee on Cancer Staging Manual (AJCC), tumor deposits (TDs) are newly included in nodal staging, which has given rise to worldwide discussions (3). AJCC 7th TNM defined TDs as discrete nodules of cancer in the pericolorectal adipose tissue's lymph drainage area of a primary carcinoma without histological evidence of residual lymph node (LN) tissue in the nodule (3). AJCC had several changes to the TD definition and category criteria in the latest four versions, which indicates TDs are controversial $(4,5)$. Both AJCC 7th TNM and AJCC 8th TNM classified any p T category lesions with negative regional LNM and positive TDs as N1c. But until now, TDs are neglected in the current TNM staging system when TDs and LN metastases (LNMs) coexist.

TDs, reported in $4.9-41.8 \%$ of patients with CRC, is an important clinical characteristic and is associated with a poor prognosis $(6,7)$. Several reports $(8-13)$ have addressed the adverse prognostic impact of TDs in CRC. However, the optimal classification of TDs in the TNM staging system has yet to be determined. Especially when TDs and LNMs coexist, clinicians always feel confused how to estimate the actual prognosis impact in consideration of TDs. Up to now, no viewpoint about the actual impact of TDs on prognosis was general accepted. This is primarily because the majority of previous studies were based on single institutional datasets that included a relatively small number of patients, making it difficult to determine the precise clinical significance of TDs by performing detailed subset analyses.

To examine the precise impact of TDs on prognosis, especially under the condition that TDs and LNMs coexist, we retrospectively analyzed two independent large cohorts of patients with CRC, the Surveillance Epidemiology and End Results (SEER) cohort and the Fudan University Shanghai Cancer Center (FUSCC) cohort, and determined

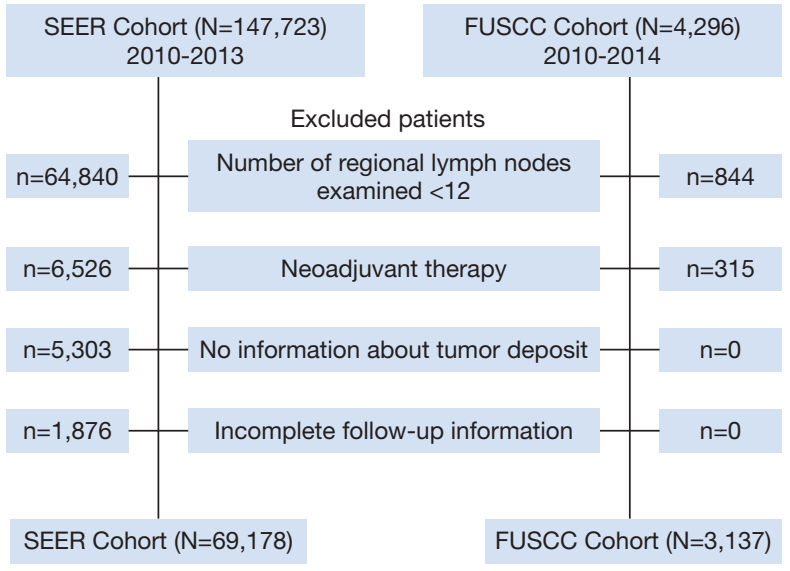

Figure 1 Consort diagram showing the exclusion criteria of the study.

the optimal classification of TDs with residual LNM in the TNM staging system.

\section{Methods}

\section{Patients}

Two independent large-scale cohorts of stage I-IV CRC patients were retrospectively analyzed. The first cohort was obtained from the SEER database. It comprised 147,723 patients with CRC who underwent curative resection between 2010 and 2013. Information on the TNM classification was retrieved based on AJCC 7th TNM.

The second cohort was obtained from the FUSCC, which comprised 4,296 CRC patients who underwent curative resection between 2010 and 2014. The diagnosis of CRC and the definition of TDs were confirmed according to AJCC 7th TNM. We reviewed all patients, and collected their clinicopathological characteristics, including age, sex, tumor location, vascular invasion, perineural invasion, number of LNMs, and the presence of TDs. Exclusion criteria included: (I) number of regional LNs examined $<12$; (II) neoadjuvant therapy; (III) no information on TDs; and (IV) incomplete follow-up data (Figure 1).

All study participants in the FUSCC cohort provided informed written consent. The protocol was approved by the Institutional Review Board Committee of the FUSCC (Shanghai, China). Research was conducted in accordance with the 1964 Declaration of Helsinki and its later amendments. The data that support the findings of this study are available on request from the corresponding author. 


\section{Statistical analyses}

All statistical analyses were conducted using Statistical Package for the Social Sciences for Windows, software version 22 (IBM Inc., Armonk, NY, USA). Continuous data are presented as the mean \pm standard deviation. The demographic and clinicopathological characteristics were analyzed using Chi-square tests for categorical variables. Overall survival (OS) was measured from the date of the initial diagnosis to the date of death or last follow-up. OS curves were analyzed using the Kaplan-Meier method, with $\mathrm{P}$ value determined by a log-rank test. Hazard ratios (HRs) and $95.0 \%$ confidence intervals (CIs) were calculated. Univariate and multivariate analyses were performed by Cox proportional hazard model. In multivariate analyses, the clinicopathological characteristics with $\mathrm{P}<0.05$ in univariate analysis were included to identify independent prognostic factors. A two-sided $\mathrm{P}<0.05$ was considered statistically significant.

\section{Results}

\section{Characteristics of CRC with TDs}

We analyzed two large cohorts, the SEER cohort and the FUSCC cohort, which included 69,178 and 3,137 patients with CRC respectively. Overall, positive TDs were observed in $12.3 \%(n=8,480)$ and $14.8 \%(n=463)$ of patients in the SEER and FUSCC cohorts, respectively. In the SEER cohort, 4,158 patients presented the precise number of TDs, and the mean number of TDs was $3.3 \pm 0.1$ (range, $1-81$ ). In the FUSCC cohort, the mean number of TDs was $2.3 \pm 0.1$ (range, $1-14$ ). Detailed clinicopathological data of the SEER cohort and the FUSCC cohort is shown in Table 1.

The incidence of TDs did not differ regarding sex, but was significantly associated with increasing $\mathrm{pT} / \mathrm{N}$ categories $(\mathrm{P}<0.001)$, PNI status $(\mathrm{P}<0.001)$, tumor location $(\mathrm{P}<0.001$ and $\mathrm{P}=0.032$ for the SEER and FUSCC cohorts, respectively) in the both cohorts. A positive correlation was also observed between the incidence of TDs and a positive VI status $(\mathrm{P}<0.001)$ in the FUSCC cohort, which was unavailable in the SEER cohort.

\section{TDs and $O S$}

We examined the relationship between several clinicopathological characteristics and OS of CRC by univariate and multivariate analysis (Tables 2,3). In the SEER cohort, both univariate and multivariate analysis showed that age, sex, tumor location, pT, pN, pM, PNI, and TDs were all associated with OS (all $\mathrm{P}<0.001$ ). In the FUSCC cohort, univariate and multivariate analysis showed the similar results with the SEER cohort, except that sex was not correlated with OS. What's more, the FUSCC cohort indicated VI was associated with OS in both univariate and multivariate analysis $(\mathrm{P}<0.001)$.

TDs exerted a significant adverse effect on the OS of all patients in the SEER (HR: 2.685, 95.0\% CI: 2.594-2.780; $\mathrm{P}<0.001$ ) and FUSCC (HR: 3.246, 95.0\% CI: 2.607-4.042; $\mathrm{P}<0.001)$ cohorts. Multivariate analysis showed TDs were independent prognostic factors for OS. With respect to LNM status, TD-positive patients exhibit an increased risk of death compared with TD-negative patients across three different $\mathrm{pN}$ categories in the SEER and FUSCC cohorts: N0 (HR: 1.72, 95.0\% CI: 1.499-1.980; P<0.001), N1 (HR: 1.69, 95.0\% CI: $1.522-1.880 ; \mathrm{P}<0.001$ ), and N2 (HR: 1.41 , 95.0\% CI: 1.290-1.538; $\mathrm{P}<0.001$ ), respectively (Figure 2 ).

\section{The unique prognosis characteristics of TDs different from LNMs}

There was a significant increase in the incidence of TDs with increasing $\mathrm{pN}$ stage in the two cohorts. To determine the significance of the number of TDs and LNMs on OS, we evaluated the prognostic impact of TDs and LNMs in two independent groups with known TD and LNM numbers: the TD-negative, LNM-positive group (TD-/ $\mathrm{LNM}+$ ) and the TD-positive, LNM-negative group (TD+/ LNM-). In the TD-/LNM+ group, increasing number of LNMs was significantly associated with an adverse OS outcome in the SEER cohort $(\mathrm{P}<0.001)$. Conversely, in the TD+/LNM- group, there were no significant differences in OS outcomes with different number of TDs in the SEER cohort $(\mathrm{P}=0.982 ;$ Figure $3 A, B)$. The same results were also found in the FUSCC cohort (Figure 3C,D).

In order to identify whether TDs was equal to LNMs with respect to survival outcome, we conducted comparison on survival between LNMs and TDs in the 2 groups (TD-/ LNM+; TD+/LNM-). The OS rates of patients with 1 TD were slightly worse than that of the patients with 1 LNM $(\mathrm{P}=0.02)$, When the number was 2 or 3 , there was no significant prognostic difference between patients with TD+LNM- and patients with TD-/LNM+ in 2 cohorts. When the number was 4 or more, OS was obviously decreased in TD-/LNM+ group compared with TD+/ LNM- group in SEER cohort $(\mathrm{P}<0.001)$ (Figure S1). 
Table 1 Baseline clinicopathological characteristics

\begin{tabular}{|c|c|c|c|c|c|c|}
\hline Characteristic & \multicolumn{3}{|c|}{ SEER $(\mathrm{N}=69,178)(\%)$} & \multicolumn{3}{|c|}{ FUSCC $(\mathrm{N}=3,137)(\%)$} \\
\hline Age, years & & & $<0.001$ & & & 0.874 \\
\hline$<65$ & 3,835 (13.8) & $23,860(86.2)$ & & $142(14.6)$ & $830(85.4)$ & \\
\hline$\geq 65$ & 4,645 (11.2) & $36,838(88.8)$ & & $321(14.8)$ & 1,844 (85.2) & \\
\hline Male & $4,246(12.4)$ & $30,018(87.6)$ & & $269(14.7)$ & $1,564(85.3)$ & \\
\hline Female & $4,234(12.1)$ & $30,680(87.9)$ & & $194(14.9)$ & $1,110(85.1)$ & \\
\hline Location & & & $<0.001$ & & & 0.032 \\
\hline Colon & 4,999 (11.9) & $39,091(88.1)$ & & 198 (13.3) & $1,290(86.7)$ & \\
\hline pT stage & & & $<0.001$ & & & $<0.001$ \\
\hline T0/Tis & $7(0.7)$ & 1,008 (99.3) & & - & - & \\
\hline $\mathrm{T} 1 / \mathrm{T} 2$ & $370(2.0)$ & $18,190(98.0)$ & & $62(8.2)$ & $694(91.8)$ & \\
\hline $\mathrm{T} 3 / \mathrm{T} 4$ & $8,094(16.4)$ & $41,386(83.6)$ & & 399 (17.2) & $1,917(82.8)$ & \\
\hline Unknown & $9(7.8)$ & $106(92.2)$ & & $2(3.1)$ & $63(96.9)$ & \\
\hline pN stage & & & $<0.001$ & & & $<0.001$ \\
\hline No & $1,715(4.2)$ & $38,828(95.8)$ & & $96(5.7)$ & $1,600(94.3)$ & \\
\hline N1 & 2,503 (15.6) & $13,535(84.4)$ & & $155(19.3)$ & $649(80.7)$ & \\
\hline Perineural invasion & & & $<0.001$ & & & $<0.001$ \\
\hline Positive & 2,165 (35.2) & $4,806(64.8)$ & & $202(30.3)$ & $464(69.7)$ & \\
\hline Negative & $5,131(9.2)$ & $50,810(90.8)$ & & $260(10.8)$ & 2,151 (89.2) & \\
\hline Unknown & 734 (12.6) & $5,082(87.4)$ & & $1(1.7)$ & 59 (98.3) & \\
\hline
\end{tabular}

SEER, Surveillance Epidemiology and End Results; FUSCC, Fudan University Shanghai Cancer Center; TD, tumor deposit.

\section{Modified pathological N category with TDs}

Then, we integrated TDs into the $\mathrm{pN}$ category of the TNM staging system on the basis of AJCC 7th TNM. This modified pN category included six classifications: N0 (no regional LNMs or TDs), N1 (metastasis in 1-3 regional LNs without TDs), N1c (TDs without regional LNM), N1TD (TDs with metastasis in 1-3 regional LNs), N2 (metastasis in $\geq 4$ regional LNs without TDs), and N2TD (TDs with metastasis in $\geq 4$ regional LNs). Survival analysis of the modified $\mathrm{pN}$ category revealed four main survival trends in the SEER cohort: (I) N0 had the longest OS time; (II) the prognosis of $\mathrm{pN1c}$ was slightly poorer than that of $\mathrm{N} 1$ (including $\mathrm{pN} 1 \mathrm{a}$ and $\mathrm{pN} 1 \mathrm{~b}$ patients) $(\mathrm{P}<0.001)$; (III) N1TD and N2 exerted similar effects on OS ( $\mathrm{P}=0.16)$; 
Table 2 Univariate and multivariate analyses of overall survival for the SEER cohort

\begin{tabular}{|c|c|c|c|c|c|c|}
\hline Prognostic factor & \multicolumn{3}{|c|}{ Univariate analysis } & \multicolumn{3}{|c|}{ Multivariate analysis } \\
\hline Age, years & $<0.001$ & 0.5 & $0.484-0.517$ & $<0.001$ & 0.412 & $0.399-0.426$ \\
\hline Sex & $<0.001$ & 0.948 & $0.921-0.976$ & $<0.001$ & 0.92 & $0.894-0.947$ \\
\hline Location & $<0.001$ & 0.82 & $0.797-0.845$ & $<0.001$ & 0.871 & $0.846-0.897$ \\
\hline $\mathrm{pN}$ stage & $<0.001$ & 1.9 & $1.868-1.933$ & $<0.001$ & 1.458 & $1.430-1.488$ \\
\hline pM stage & $<0.001$ & 3.989 & $3.873-4.109$ & $<0.001$ & 3.047 & 2.939-3.159 \\
\hline Perineural invasion & $<0.001$ & 1.128 & $1.112-1.145$ & $<0.001$ & 1.03 & $1.014-1.047$ \\
\hline Tumor deposit & $<0.001$ & 2.685 & $2.594-2.780$ & $<0.001$ & 1.411 & $1.358-1.465$ \\
\hline
\end{tabular}

SEER, Surveillance Epidemiology and End Results; HR, hazard ratio; Cl, confidence interval.

Table 3 Univariate and multivariate analyses of overall survival for the FUSCC cohort

\begin{tabular}{|c|c|c|c|c|c|c|}
\hline Prognostic factor & \multicolumn{3}{|c|}{ Univariate analysis } & \multicolumn{3}{|c|}{ Multivariate analysis } \\
\hline Age, years & $<0.001$ & 0.654 & $0.529-0.809$ & $<0.001$ & 0.514 & $0.412-0.641$ \\
\hline Sex & 0.354 & 1.104 & $0.895-1.362$ & - & - & - \\
\hline Location & 0.01 & 0.765 & $0.625-0.937$ & 0.041 & 0.805 & $0.654-0.992$ \\
\hline pN stage & $<0.001$ & 2.468 & $2.175-2.801$ & $<0.001$ & 1.878 & $1.620-2.178$ \\
\hline pM stage & $<0.001$ & 1.781 & $1.509-2.102$ & $<0.001$ & 1.978 & $1.603-2.440$ \\
\hline Venous invasion & $<0.001$ & 1.685 & $1.508-1.883$ & $<0.001$ & 1.492 & $1.273-1.749$ \\
\hline Perineural invasion & $<0.001$ & 1.493 & $1.304-1.710$ & 0.009 & 1.357 & $1.079-1.707$ \\
\hline
\end{tabular}

FUSCC, Fudan University Shanghai Cancer Center; HR, hazard ratio; Cl, confidence interval.

and (IV) N2TD had the shortest OS, with much worse prognosis than $\mathrm{N} 2(\mathrm{P}<0.001$, Figure $4 A)$. Similar survival characteristics were observed in the FUSCC cohort, except that there were no significant differences in the prognostic impact of $\mathrm{N} 1$ and $\mathrm{N} 1 \mathrm{c}(\mathrm{P}=0.15$, Figure $4 B)$. In accord with the SEER cohort, the FUSCC also suggested N1TD and $\mathrm{N} 2$ had similar OS $(\mathrm{P}=0.23)$ and N2TD exhibited worst prognosis (Figure 4B).

\section{Discussion}

The TNM staging system has been the benchmark on tumor staging and played a key role in cancer treatment.
TDs were first introduced in the fifth edition of TNM staging system, which adopted the $3.0-\mathrm{mm}$ rule to classify TDs (4). In the sixth and seventh editions of the TNM staging system, the classification criteria of TDs were changed significantly $(3,5)$. Seventh edition of the TNM staging system first included TDs into TNM systems and introduced a new concept, named as $\mathrm{pN} 1 \mathrm{c}$, which has been defined as any $\mathrm{pT}$ lesion with TDs but lacking regional LNMs (3). The pN1c classification seemed reasonable given the reliability of the clinical evidence obtained from several studies (14-18). In the eighth edition of the TNM staging system, the $\mathrm{pN} 1 \mathrm{c}$ classification has remained unchanged. However, in the current TNM staging system, 

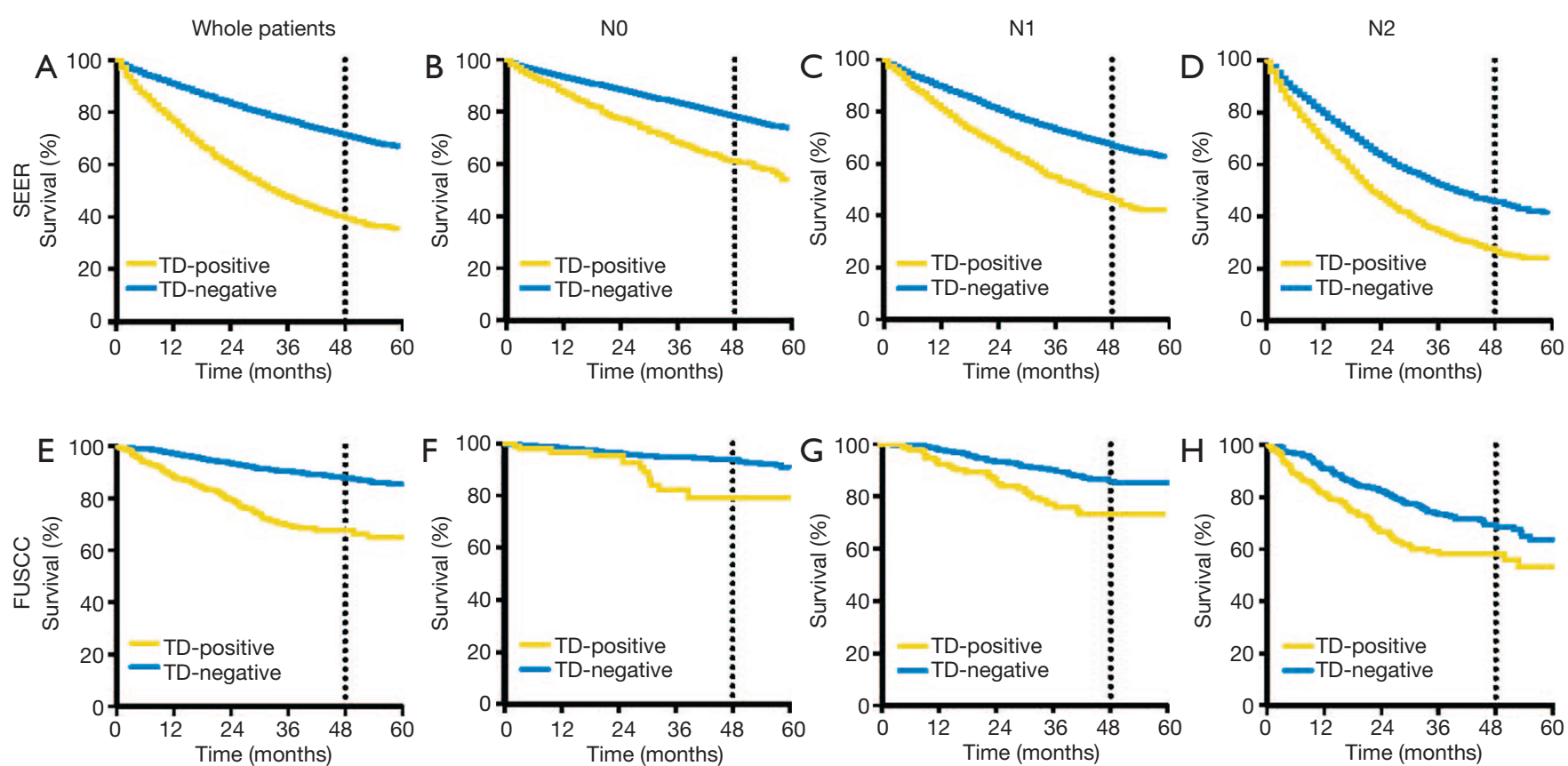

Figure 2 Kaplan-Meier curves of TDs status in overall patients and different N stage patients in the SEER and FUSCC database. (A,B,C,D) The SEER cohort: (A) TD-positive patients exerted poorer prognosis in overall patients $(\mathrm{P}<0.001)$; (B,C,D) TD-positive patients exerted poorer prognosis in N0, N1, N2 patients respectively (all $\mathrm{P}<0.001$ ). (E,F,G,H) The FUSCC cohort: (E) TD-positive patients exerted poorer prognosis in overall patients $(\mathrm{P}<0.001)$; $(\mathrm{F}, \mathrm{G}, \mathrm{H}) \mathrm{TD}$-positive patients exerted poorer prognosis in N0, N1, N2 patients respectively (all $\mathrm{P}<0.001)$. TD, tumor deposit; SEER, Surveillance Epidemiology and End Results; FUSCC, Fudan University Shanghai Cancer Center.

when LNMs were positive, the presence of TDs was not considered. The confusing role of TDs in the TNM staging system may be due to lacking strong and powerful evidence to confirm more detail prognostic characteristic of TDs in CRC patients.

The adverse prognostic impact of TDs in CRC seems to reach a consensus, which have addressed by a number of researches (8-13). Nevertheless, how to classify TDs in the TNM staging system is still controversial. Classifying TDs as LNMs was one potential solution to solve this problem. $\mathrm{Li}$ et al. (19) proposed that the classification of TDs as LNMs was potentially superior to the classification in the seventh edition of the TNM staging system. Nagtegaal et al. (6) concluded that TDs should not be classified as LNMs because the origins of TDs are so diverse (perineural/ perivascular/intravascular), but they also suggested to add the number of TDs to the number of LNMs. However, Frankel et al. (20) speculated that the number of TDs should not be combined with the total number of positive LNMs and that TDs should only be considered in the presence of LNMs, which totally agreed with the current TNM staging system. Basnet et al. (21) ascertained that TDs are not equal to LNMs, but didn't give more information for the precise role of TDs in the TNM system. Mayo et al. (22) found that TDs were associated with poor prognosis in patients of any $\mathrm{N}$ categories. Mirkin et al. (23) showed that patients with TDs alone had similar prognosis to who with LNMs alone in stage III colon cancer. These results supported that TDs are different from LNMs and may have unique prognostic characteristics.

In the present study, we retrospectively analyzed two independent large-scale cohorts of CRC patients from SEER database and FUSCC, involving a total of 72,315 patients. Our results showed that TDs in patients with CRC had unique prognostic characteristics. Whether LNMs were present or not, TDs were an independent adverse prognostic factor for OS, which was testified in the two cohorts. The incidence of TDs was significantly associated with LNMs $(\mathrm{P}<0.001)$, but TDs had different prognostic pattern compared with LNMs. We found that the prognosis worsened as the number of LNMs increased. Remarkably, the presence of TDs was associated with OS, but the number of TDs had no effect on prognosis. The prognosis of patients with TDs was not exactly same as 

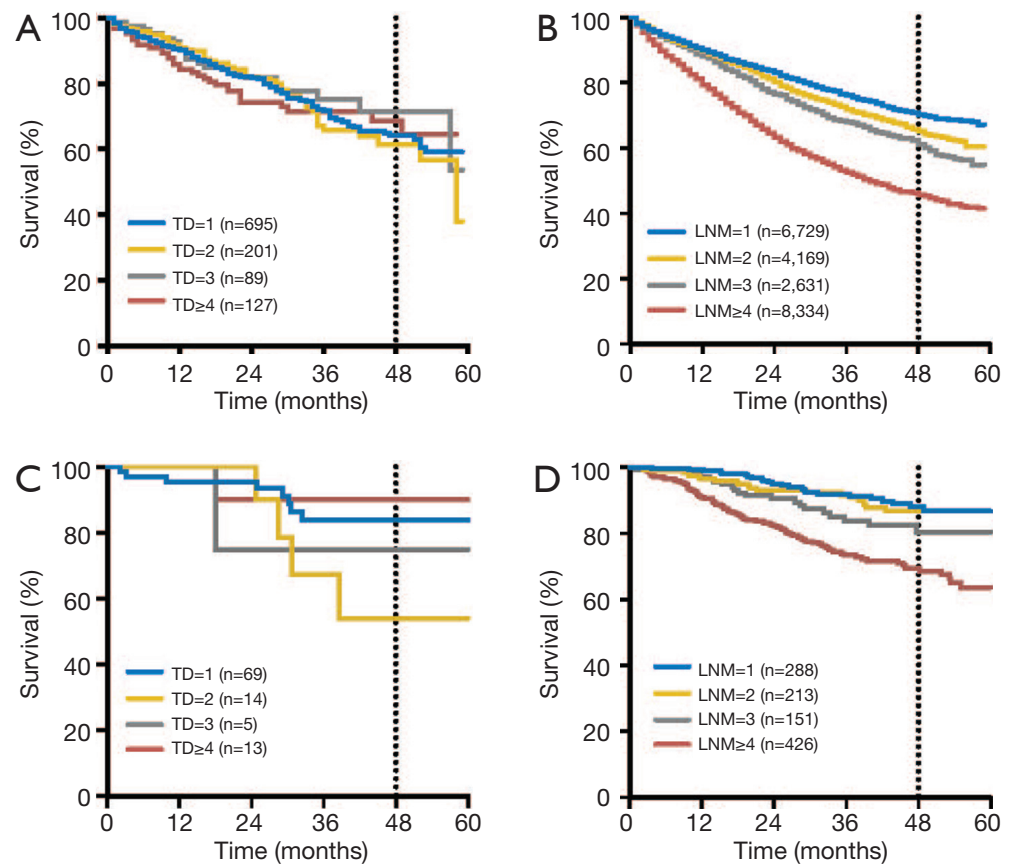

Figure 3 Respective prognosis characteristic of tumor deposits and lymph node metastasis on number. (A,B) In the SEER cohort. (A) There were no significant differences in OS outcomes with increasing number of TDs ( $\mathrm{P}=0.982)$. (B) Increasing number of LNMs were significantly associated with an adverse OS outcome $(\mathrm{P}<0.001)$. (C,D) In the FUSCC cohort. (C) There were no significant differences in OS outcomes with increasing number of TDs $(\mathrm{P}=0.252)$. (D) Increasing number of LNMs were significantly associated with an adverse OS outcome $(\mathrm{P}<0.001)$. OS, overall survival; TD, tumor deposit; SEER, Surveillance Epidemiology and End Results; FUSCC, Fudan University Shanghai Cancer Center.
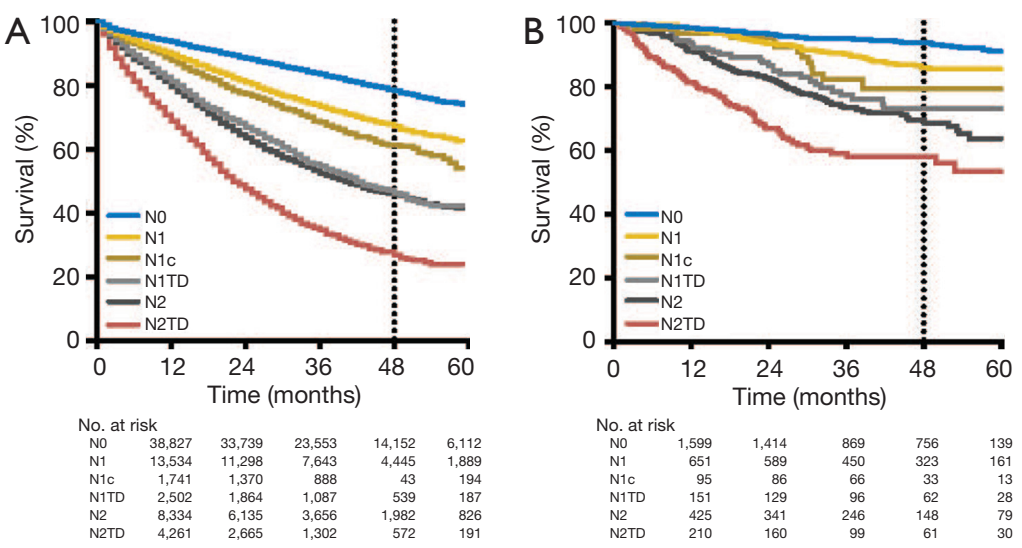

Figure 4 Kaplan-Meier curves of modified pathological N category with tumor deposits. (A) In the SEER cohort, survival analysis of the modified pN category revealed four main survival trends: (I) N1 and N1c exerted similar effects on OS, although the prognosis of N1c was slightly poorer than that of $\mathrm{pN} 1$ (including N1a and N1c patients) ( $\mathrm{P}<0.001$ ); (II) N1TD and N2 exerted similar effects on OS (P=0.16); (III) N0 exhibited the longest OS times; and (IV) N2TD exhibited the poorest OS times. (B) The above results were validated in the FUSCC cohort: (I) there were no significant differences in the prognostic impact of N1 and N1c ( $\mathrm{P}=0.15)$. (II) N1TD and N2 exerted similar effects on OS ( $\mathrm{P}=0.23$ ). (III) N0 exhibited the longest OS times; and (IV) N2TD exhibited the poorest OS times. OS, overall survival; TD, tumor deposit; SEER, Surveillance Epidemiology and End Results; FUSCC, Fudan University Shanghai Cancer Center. 
the one with same number of LNMs, especially when the number was bigger than 4 . The difference in prognosis indicated that TDs should not be classified as LNMs. Regarding combining TDs with LNMs, our results testified that $\mathrm{N} 1 \mathrm{c}$ has poorer OS than $\mathrm{N} 0$ and has similar prognosis with $\mathrm{N} 1$, which indicates N1c category defined by AJCC $7^{\text {th }}$ and $8^{\text {th }}$ TNM systems indeed makes sense. However, in the presence of LNMs, the prognosis of patients with N1TD (N1 with positive TDs) was same as the $\mathrm{N} 2$ patients, and N2TD (N2 with positive TDs) patients had much worse prognosis than N2. These finds indicate we shouldn't ignore the presence of TDs when LNMs are positive, but this situation has just been neglected in AJCC $7^{\text {th }}$ and $8^{\text {th }}$ TNM systems.

Based on these findings, we speculate that TDs may be integrated into the $\mathrm{pN}$ category of the TNM staging system, with this modified version of the $\mathrm{pN}$ category taking into consideration the existence but not the number of TDs. This may be a reasonable solution for the postoperative staging of patients in whom TDs and LNMs occur simultaneously. This modified $\mathrm{pN}$ category was also applicable to the popular topic about three versus six months adjuvant oxaliplatin-based chemotherapy for patients with stage III colon cancer. For the low-risk group patients, including $\mathrm{T} 1-3$ and N1, three months adjuvant oxaliplatin-based chemotherapy was non-inferior than sixmonths $(24,25)$. However, for the high-risk group patients, including $\mathrm{T} 4$ and $\mathrm{N} 2$, six months adjuvant treatment was superior to three months treatment $(24,25)$. Our results showed that the prognosis of CRC patients with $\mathrm{N} 1$ and TDs was similar with N2 patients'. Therefore, for patients with N1 with TDs, they were transformed from low-risk group into high-risk group which need a longer period of treatment.

To our knowledge, this study is the largest research about TDs' prognostic role in CRC to date, which could provide a reference for the modern TNM staging system. Even so, our study has several limitations. First, this is a large-scale retrospective study, which lacks strict experimental design and might yield selection bias. However, our sample size is big enough to cushion the selection bias and the data from the two independent cohorts reflect our clinical practice in the real world. Secondly, size, contour and distribution of TDs were ignored in our analysis. In view of the insufficient clinical data and complicated detection procedures for these features of TDs, we only focused on the number of TDs. These features are needed to be further explored.

\section{Conclusions}

In conclusion, our results suggest that TDs had unique prognosis characteristic different from LNMs in regard to number, and we should not ignore TDs in the presence of positive LNMs. We propose that, in future TNM staging system, TDs should be integrated into the $\mathrm{pN}$ category whether in the absence or presence of LNMs, taking into consideration the existence but not the number of TDs. This modified pN category may offer more precise prognosis and be useful to choose suitable period of adjuvant treatment.

\section{Acknowledgments}

Funding: This research was supported in part by the Natural Science Foundation of Shanghai (16ZR1406700), the National Natural Science Foundation of China (81472620), and Shanghai Municipal Commission of Health and Family Planning (2014ZYJB0101).

\section{Footnote}

Conflicts of Interest: The authors have no conflicts of interest to declare.

Ethical Statement: The authors are accountable for all aspects of the work in ensuring that questions related to the accuracy or integrity of any part of the work are appropriately investigated and resolved. All study participants in the FUSCC cohort provided informed written consent. The protocol has been approved by the Institutional Review Board Committee of the FUSCC (Shanghai, China) and the work has been conducted in accordance with the 1964 Declaration of Helsinki and its later amendments. The data that support the findings of this study are available on request from the corresponding author.

\section{References}

1. Chen W, Zheng R, Baade PD, et al. Cancer statistics in China, 2015. CA Cancer J Clin 2016;66:115-32.

2. Zeng H, Chen W, Zheng R, et al. Changing cancer survival in China during 2003-15: a pooled analysis of 17 population-based cancer registries. Lancet Glob Health 2018;6:e555-67. 
3. Edge SB, Compton CC. The American Joint Committee on Cancer: the 7th edition of the AJCC cancer staging manual and the future of TNM. Ann Surg Oncol 2010;17:1471-4.

4. Sobin LH, Fleming ID. TNM Classification of Malignant Tumors, fifth edition (1997). Union Internationale Contre le Cancer and the American Joint Committee on Cancer. Cancer 1997;80:1803-4.

5. Sobin LH. TNM, sixth edition: new developments in general concepts and rules. Semin Surg Oncol 2003;21:19-22.

6. Nagtegaal ID, Knijn N, Hugen N, et al. Tumor Deposits in Colorectal Cancer: Improving the Value of Modern Staging-A Systematic Review and Meta-Analysis. J Clin Oncol 2017;35:1119-27.

7. Lord AC, D'Souza N, Pucher PH, et al. Significance of extranodal tumour deposits in colorectal cancer: A systematic review and meta-analysis. Eur J Cancer 2017;82:92-102.

8. Belt EJ, van Stijn MF, Bril H, et al. Lymph node negative colorectal cancers with isolated tumor deposits should be classified and treated as stage III. Ann Surg Oncol 2010;17:3203-11.

9. Tong LL, Gao P, Wang ZN, et al. Is the seventh edition of the UICC/AJCC TNM staging system reasonable for patients with tumor deposits in colorectal cancer? Ann Surg 2012;255:208-13.

10. Yamano T, Semba S, Noda M, et al. Prognostic significance of classified extramural tumor deposits and extracapsular lymph node invasion in T3-4 colorectal cancer: a retrospective single-center study. BMC Cancer 2015;15:859.

11. Ueno H, Hashiguchi Y, Shimazaki H, et al. Peritumoral deposits as an adverse prognostic indicator of colorectal cancer. Am J Surg 2014;207:70-7.

12. Nagayoshi K, Ueki T, Nishioka Y, et al. Tumor deposit is a poor prognostic indicator for patients who have stage II and III colorectal cancer with fewer than 4 lymph node metastases but not for those with 4 or more. Dis Colon Rectum 2014;57:467-74.

13. Nagtegaal ID, Tot T, Jayne DG, et al. Lymph nodes, tumor deposits, and TNM: are we getting better? J Clin Oncol 2011;29:2487-92.

14. von Winterfeld M, Hoffmeister M, Ingold-Heppner $\mathrm{B}$, et al. Frequency of therapy-relevant staging shifts in colorectal cancer through the introduction of $\mathrm{pN} 1 \mathrm{c}$ in the 7th TNM edition. Eur J Cancer 2014;50:2958-65.
15. Prabhudesai A, Arif S, Finlayson CJ, et al. Impact of microscopic extranodal tumor deposits on the outcome of patients with rectal cancer. Dis Colon Rectum 2003;46:1531-7.

16. Nagtegaal ID, Quirke P. Colorectal tumour deposits in the mesorectum and pericolon; a critical review. Histopathology 2007;51:141-9.

17. Goldstein NS, Turner JR. Pericolonic tumor deposits in patients with $\mathrm{T} 3 \mathrm{~N}+\mathrm{MO}$ colon adenocarcinomas: markers of reduced disease free survival and intra-abdominal metastases and their implications for TNM classification. Cancer 2000;88:2228-38.

18. Tateishi S, Arima S, Futami K, et al. A clinicopathological investigation of "tumor nodules" in colorectal cancer. Surg Today 2005;35:377-84.

19. Li J, Yang S, Hu J, et al. Tumor deposits counted as positive lymph nodes in TNM staging for advanced colorectal cancer: a retrospective multicenter study. Oncotarget 2016;7:18269-79.

20. Frankel WL, Jin M. Serosal surfaces, mucin pools, and deposits, oh my: challenges in staging colorectal carcinoma. Mod Pathol 2015;28 Suppl 1:S95-108.

21. Basnet S, Lou QF, Liu N, et al. Tumor deposit is an independent prognostic indicator in patients who underwent radical resection for colorectal cancer. J Cancer 2018;9:3979-85.

22. Mayo E, Llanos AA, Yi X, et al. Prognostic value of tumour deposit and perineural invasion status in colorectal cancer patients: a SEER-based population study. Histopathology 2016;69:230-8.

23. Mirkin KA, Kulaylat AS, Hollenbeak CS, et al. Prognostic Significance of Tumor Deposits in Stage III Colon Cancer. Ann Surg Oncol 2018;25:3179-84.

24. Iveson TJ, Kerr RS, Saunders MP, et al. 3 versus 6 months of adjuvant oxaliplatin-fluoropyrimidine combination therapy for colorectal cancer (SCOT): an international, randomised, phase 3, non-inferiority trial. Lancet Oncol 2018;19:562-78.

25. Grothey A, Sobrero AF, Shields AF, et al. Duration of Adjuvant Chemotherapy for Stage III Colon Cancer. N Engl J Med 2018;378:1177-88.

Cite this article as: Liu F, Zhao J, Li C, Wu Y, Song W, Guo T, Chen S, Cai S, Huang D, Xu Y. The unique prognostic characteristics of tumor deposits in colorectal cancer patients. Ann Transl Med 2019;7(23):769. doi: 10.21037/atm.2019.11.69 

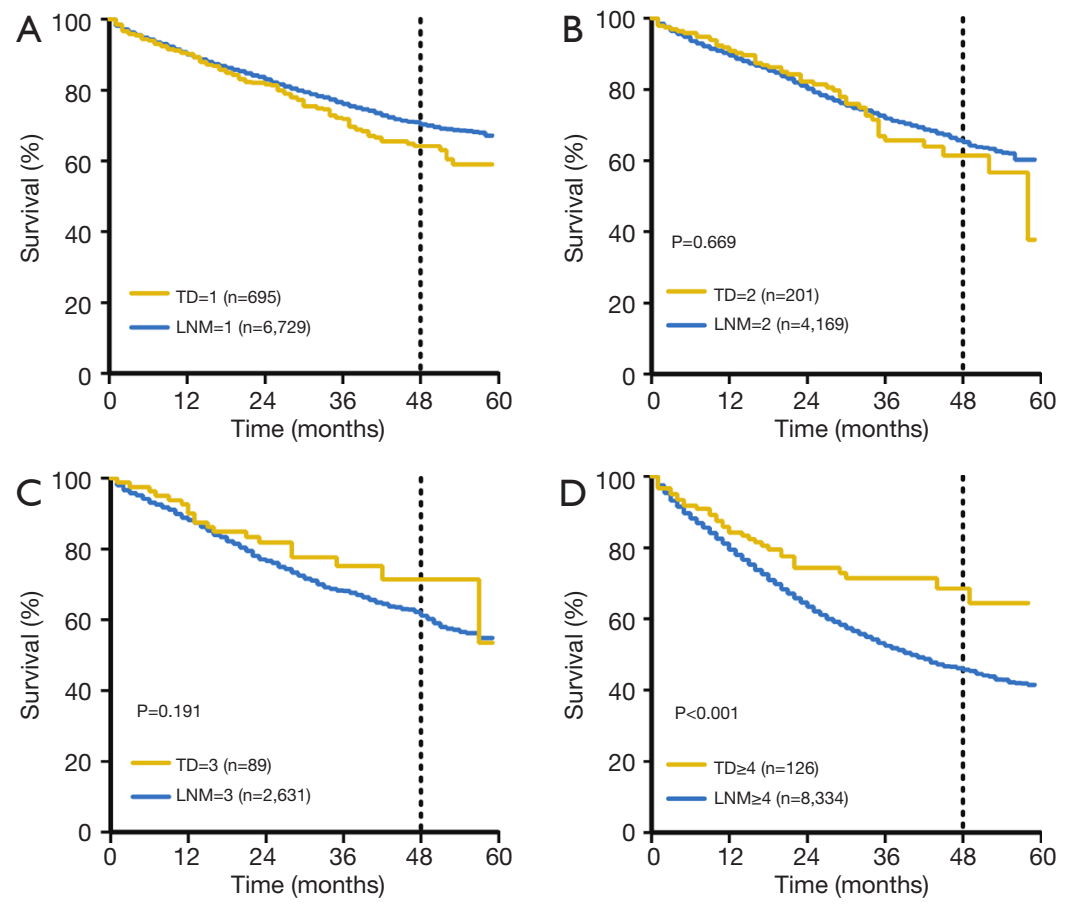

Figure S1 Comparison on survival between LNMs and TDs in the 2 groups (TD-LN+; TD+LN-) in the SEER cohort. (A) The OS outcomes of patients with a single TD were slightly poorer than those of patients with a single $\mathrm{LNM}(\mathrm{P}=0.02)$. (B,C) When the number was 2 or 3, there were no significant prognostic differences between TD-positive, LNM-negative and TD-negative, LNM-positive patients in the two cohorts $(\mathrm{P}>0.05)$. (D) When the number was $\geq 4$, there was a significant reduction in the OS of TD-negative, LNM-positive patients compared to TD-positive, LNM-negative patients $(\mathrm{P}<0.001)$. OS, overall survival; LNM, lymph nodes metastasis; SEER, Surveillance Epidemiology and End Results. 